\title{
Development of a hybrid algorithm for time resolved stellar photometry from space images
}

\author{
Kleber Iguchi, Vanderlei C. Parro, Leonardo Pinheiro da Silva, Fabio de Oliveira Fialho, Hae Yong Kim
}

\begin{abstract}
The CoRoT satellite, inserted in a low Earth polar orbit, is exposed to a radioactive environment that affects the instrumental components. Effects of charged particle impacts on the onboard electronics and optics have been minimized by the use of radiation-hard components and proper data reduction techniques. However, when the satellite crosses the South Atlantic Anomaly, the CCDs are exposed to up to 10 minutes of strong radiation, subjecting up to $9 \%$ of the whole satellite's imaging duty cycle to some kind of impact damage. This work presents an innovative hybrid photometry algorithm that takes advantage of a high-resolution instrumental point spread function and of the high signal-to-noise-ratio obtained in the onboard aperture photometry, to extract a more accurate photometric signal from the observed stars. It also takes into account the total standard deviation in each pixel, formed by Poissonian and readout noises. This methodology has proven to achieve gains in photometric precision and increase the duty cycle up to $97 \%$, enabling robust and accurate data exploitation.
\end{abstract}

Keywords - Stellar Photometric Reduction, Hybrid Algorithm, SF-fitting, Optimal Aperture.

\section{INTRODUCTION}

CoRoT (Convection, Rotation and planetary Transit) is a space mission led by the Centre National d'Études Spaciales (CNES), in association with the European Space Agency (ESA) and several French laboratories, and contributions from Austria, Belgium, Brazil, Germany and Spain. The space telescope was launched in Dec. 27th, 2006 and is dedicated to stellar seismology and to the search for exoplanets.

Stellar seismology is the study of the oscillation modes of a star. Observations of stars other than the Sun provide data to refine and validate theoretical models of stellar structure and evolution, which is the main goal of the Asteroseismology program (AS). The Planet Finding program (PF) is dedicated to detecting extrasolar planets by the transit method, i.e., measuring faint, periodic achromatic decreases in the stellar flux, when a planet crosses in front of the host star. Both scientific programs require long, uninterrupted observations with high precision photometry, only possible from the space.

An overview of the literature indicates three main philosophies of data reduction to perform time-resolved stellar photometry, each with its own benefits and drawbacks: threshold, aperture and PSF-fitting algorithms. Threshold photometry presents low performance for the CoRoT [1] and is not taken into account in this paper.

This work proposes an innovative hybrid photometry algorithm that takes advantage of a high-resolution instrumental point spread function (PSF) and of the high signal-to-noise-ratio (SNR) obtained in the on-board aperture photometry, to extract a more accurate photometric signal from the observed stars. In the process of hybrid image formation and data reduction, the total standard deviation in each pixel formed by Poissonian and readout noises - is also accounted for.

This paper is organized as follows: Section II presents an overview of the instrument and considers some of its electronic and optical aspects. Section III discusses the environmental conditions, focusing on the impacts of the SAA to the distinct photometric reduction algorithms, which are described in Section IV. Section V discusses the results and Section VI finally presents the conclusions to this work.

\section{THE SATELLITE}

The CoRoT satellite is a $626 \mathrm{~kg}, 4.1 \mathrm{~m}$ long spacecraft based on a PROTEUS platform, designed for low Earth orbit missions by CNES and Thales-Alenia Space (former Alcatel Space Industry). Fig. 1 depicts the satellite, which can be divided in four main components, as follows [1]:

- an afocal telescope composed of two parabolic mirrors and a baffle to minimize straylight coming from Earth, providing a field of view of $2.7^{\circ}$ by $3.05^{\circ}$;

- a wide-field camera composed of a dioptric objective, and a focal plane assembly consisting of four CCD detectors and a bi-prism (placed in front of the PF detectors). On the AS side, the CCDs are defocused, to avoid saturation of the brightest stars. The CCDs are frame-transfer, thinned, back-illuminated designs in a 2048x4096 pixels array. Each pixel is $13.5 \times 13.5 \mu \mathrm{m} 2$ in size, which corresponds to an angular pixel size of 2.32 arcsec;

- an equipment bay which houses CCD readout electronics, attitude control system, and digital data processing units, as well as housekeeping electronics (such as for CCD fine thermal control);

- the PROTEUS platform itself, which provides the resources for the basic (yet complex) functions of the satellite once in orbit, such as electric power supply from the solar panels, trajectory control system, and communications with ground segment through an antenna, among other functions.

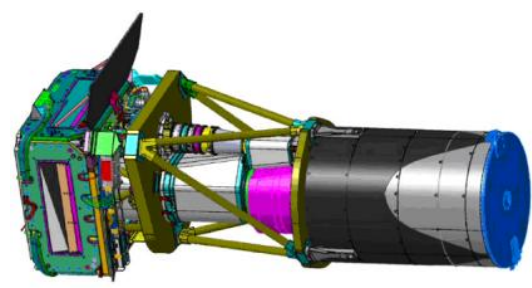

Fig. 1. Rendered image of the CoRoT telescope, camera and equipment bay (CNES).

Kleber Iguchi, Leonardo Pinheiro da Silva, Fabio de Oliveira Fialho, Hae Yong Kim, Departamento de Engenharia Elétrica, Escola Politécnica, Universidade de São Paulo, Brasil. Vanderlei Cunha Parro, Instituto Mauá de Tecnologia, São Caetano do Sul, Brasil. E-mails: kleber.iguchi@ gmail.com, vparro@maua.br, pinheirodasilva@gmail.com,f_fialho@hotmail.com, hae@lps.usp.br 


\section{The South AtLantic ANOMALY}

The CoRoT telescope was launched into a polar orbit at an average altitude of $900 \mathrm{~km}$ and period of $6184 \mathrm{~s}$ (103min). Under these conditions, the satellite is subjected to perturbations in its attitude (mainly due to Earth's gravity and magnetic fields - see Fig. 2) and to temperature variations (caused by the transition light-penumbra-shadow-penumbralight along its trajectory, and by Sun/Earth infrared emissions). Attitude jitter produces perturbation in the extracted light curves; so do the Earth albedo and the presence of debris and/or other satellites in the CoRoT's field of view.

The South Atlantic Anomaly (SAA), however, is the source of the most significant contribution to the reduction of the satellite's operational duty cycle. SAA is the region where the Earth's inner radiation belt makes its closest approach to the planet's surface. Satellites and other spacecraft passing through this region of space actually enter the Van Allen radiation belt and are bombarded by protons that may exceed energies of 100 $\mathrm{MeV}$ at a rate of 3000 'hits' per square centimeter per second. This can produce 'glitches' in astronomical data, problems with the operation of onboard electronic systems, and premature aging of computer, detectors and other spacecraft components.
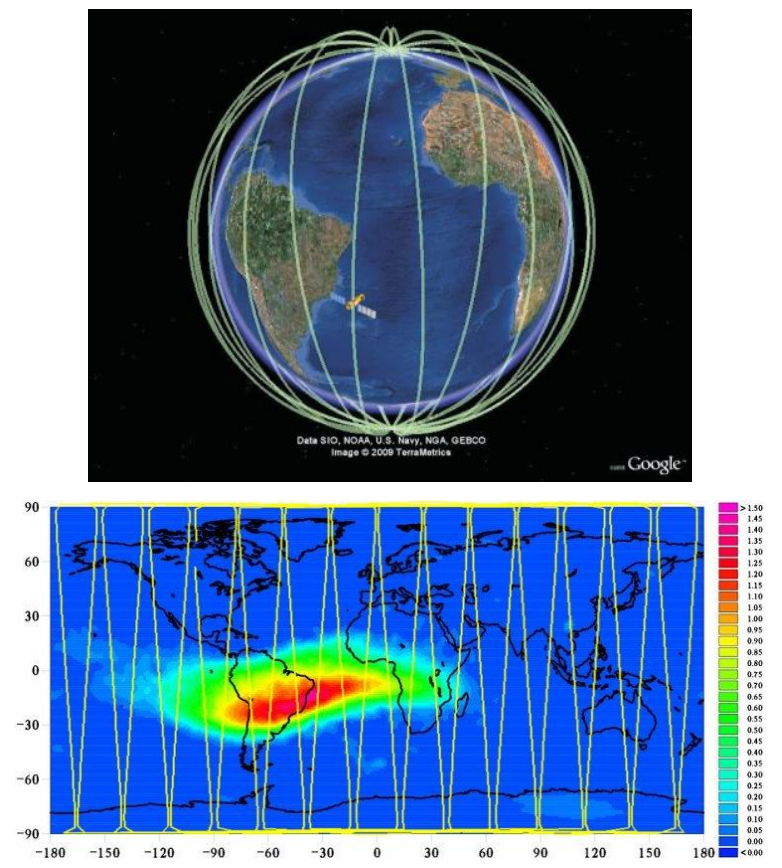

Fig. 2. Two illustrations showing a 24-hours CoRoT's trajectory, crossing 8 times the SAA in the period. (Upper image source: Terrametrics / Google Earth. Lower image source: CNES/CLS. Satellite Tracker Script: Robert Simpson, Cardiff University.)

The effects of the interaction between energetic particles and the lenses are almost limited to fluorescence, leading to a very small contribution to the mean background level. The effects on the electronics are somewhat minimized by the use of radiation-hard components.

The effects of charged particle impacts on CCDs, however, may interfere in the photometric curves in two different ways: transient artifacts on the acquired images, created by eventual $e-h$ pairs, and permanent damage due to non-ionizing interactions. The focal plane is shielded with $10 \mathrm{~mm}$ of aluminum, which is enough to stop almost the whole population of trapped electrons, and protons below $50 \mathrm{MeV}$ (Pinheiro da Silva et al., 2006). Nevertheless, when crossing the SAA ( 8 to 10 passages per day), the CCDs are exposed to up to 10 minutes of strong radiation, which means up to $9 \%$ of the whole satellite's duty cycle may be lost.

\section{METHODOLOGY}

In this scenario, in order to achieve a high duty cycle operation, the telescope must be provided with effective photometric algorithms, so the data collected during the passages through the SAA can be processed and exploited.

\section{A. Aperture Photometry}

Each star image acquired by the CCDs is composed of foreground and background pixels, i.e., the source pixels, and the surrounding pixels. The foreground pixels are then classified as central or wing pixels, according to their position in the star image.

Aperture photometry (see Fig. 3 below) defines a mask which represents the foreground pixels to be summed up in the computation of the collected photon flux for a given star. The mask represents mainly the central pixels.
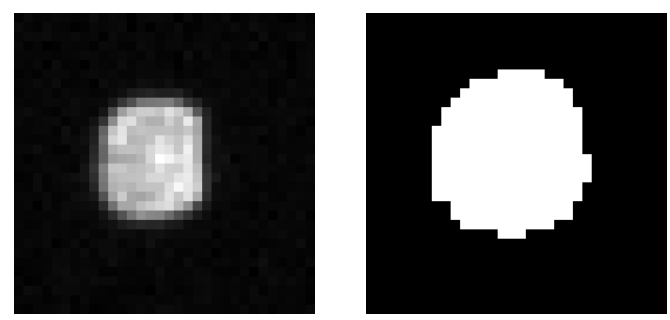

Fig. 3. Examples of an observed star (left) and of an aperture mask (right).

This method presents very high precision for isolated, bright stars and for stable satellite attitude (low jitter) scenarios. It is the data reduction technique implemented onboard, due both to link capacity constraints (given the large number of targets simultaneously observed by the instrument) and to its deterministic algorithm [2].

For each observed star in the AS program (or set of stars, in the PF program), an optimum mask is defined in order to maximize its photometric SNR, in the following steps, detailed in [3]:

- an empirical super-resolved PSF is previously estimated;

- the PSF's brighter pixels are considered to be the most significant contributors to the flux measurement. The pixels are thus sorted by decreasing intensity in an array $N_{*}^{s}$, to which is associated a set of $k$ apertures $A p(k)$ composed of the $k$ brightest pixels in the image (the first $k$ elements of $N_{*}^{s}$ );

- the photometric SNR is calculated as a CCD equation-like function of $A p(k)$, in the form

$$
\operatorname{SNR}(k)=\frac{\sum_{i=1}^{k} N_{*}^{s}(i)}{\sqrt{\sum_{i=1}^{k}\left(N_{*}^{s}(i)+\mu_{s k y}+\sigma_{i}^{2}\right)}}
$$

where $\mu_{s k y}$ is the background noise, and $\sigma_{i}^{2}$ is the readout noise per pixel. 
Eq.(1) gives a SNR characteristic growth curve, as a function of $k$, which presents a maximum at a given aperture $A p\left(k_{m}\right)$ : too few source pixels in smaller apertures and too many noisy pixels in bigger apertures degrade the photometric SNR as one moves away from this maximum [4]. An example of SNR growth curve as a function of the number of pixels included in the aperture mask is given in Fig. 4.

\section{B. PSF Fitting Photometry}

Fitting photometry allows restoration of degraded stellar images through deconvolution processes, using the empirical PSF of the optical instrument itself.

As in the aperture methodology, an empirical superresolved PSF model is reconstructed from the sequence of CCD images from the observed star, using Landweber's iterative inverse filtering technique [5]. Information about the trajectory of the star's baricenter, deduced from the telemetry data, is also used in the PSF reconstruction, as described in [6].

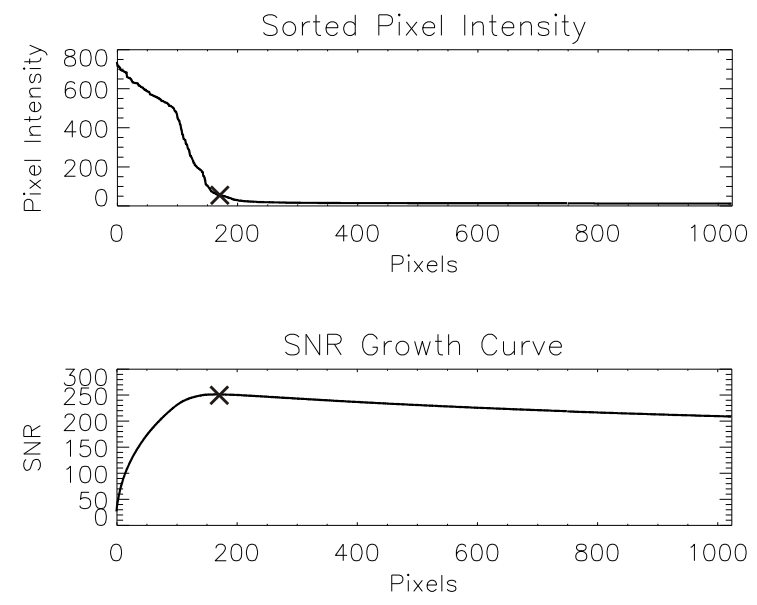

Fig. 4. SNR growth curve for simulated data. For this particular simulated star, the $k_{m}=165$ brightest pixels belong to the optimal aperture mask.

This PSF model is then recursively fitted to each image in the sequence, in a procedure based on an outlier-rejectionoriented implementation of the Levenberg-Marquardt algorithm, as detailed in [5].

Fig. 5 illustrates how accurate photometric data can be extracted from impacted images, allowing partial exploitation of the images acquired by the satellite while crossing the SAA.
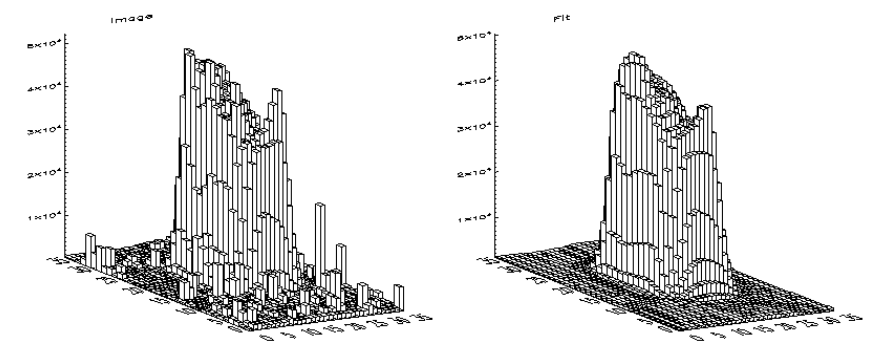

Fig. 5. Comparison between impacted observed star image (left) and the corresponding fitted PSF model (right). (Pinheiro da Silva et al., 2006).

This technique presents better performance for crowded fields and for faint stars (Fig. 6); it also presents robustness in the presence of disturbances such as straylight or high satellite attitude jitter. It is the method used by the ground team to reduce data from the images sent via telemetry, along with the aperture photometry already computed onboard.

\section{Hybrid Photometry}

One obvious flaw with the aperture method is that it does not include all the flux from a source: since the number of pixels in the aperture mask is rather small in this method, the light flux contained within the star wings is not accounted for. Therefore, not all the available information in the sequence of images is really exploited. Another flaw in this method is that when a charged particle impacts the CCD within the aperture mask, the outlier pixel generated will be accounted for as part of the star. For that reason, there's no means to tell if the light comes from the star or from an impact. Finally, there's the problem of attitude jitter: if the jitter is large enough, it will cause the target star to project its light outside the edges of the aperture mask, creating "valleys" in the extracted light curve, worsening the global SNR.

On the other hand, the recursive PSF fitting algorithm replaces all the pixels from the acquired images with their corresponding fitted values, whether their individual SNR is high or not, which might reduce the overall photometric SNR.
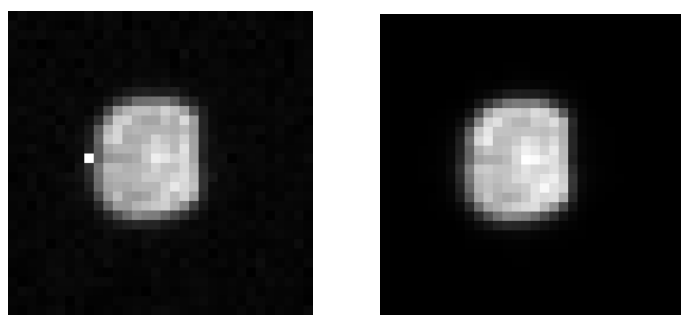

Fig. 6. Examples of an impacted image (left, where the energy imparted from a charged particle hitting the CCD causes a brighter pixel) and its PSFfitted correspondent, free from impact effect, but subject to reduction in photometric SNR, due to the very process of fitting.

This work presents an innovative hybrid photometry algorithm that takes advantage of a high-resolution instrumental PSF and of the high SNR obtained in the onboard aperture photometry, in order to extract a more accurate photometric signal from the observed stars.

The inputs to the hybrid algorithm are the sequence of acquired images, and background and readout noise levels. The jitter is then estimated from the star's centroid position over time. Using this information it is possible to calculate an empiric high-resolution PSF for the target star, from which an optimal mask is obtained. This mask will contain only highSNR pixels, which provide the best overall SNR in the photometric curve (as exposed previously).

Original low-SNR pixels will be replaced by their equivalent low-uncertainty pixels in the PSF. Besides, original impacted pixels will be replaced by modeled pixels from the PSF itself, so very impacted images can provide useful data to the light flux. These outlier pixels are detected as follows:

where

$$
Y_{D O L}=\left(Y_{i}-X_{F I T}\right) \geq n_{\sigma} \cdot \sqrt{X_{F I T}+\eta^{2}}
$$

$Y_{D O L}$ : outlier "mask" for the acquired image $Y_{i}$ ('1' for outlier, '0' for not-outlier, pixel-wide); 
$X_{F I T}$ : PSF-fitting of $Y_{i}$;

$n_{\sigma}:$ empiric parameter for $\sigma$;

$\eta \quad$ : total noise associated to each pixel.

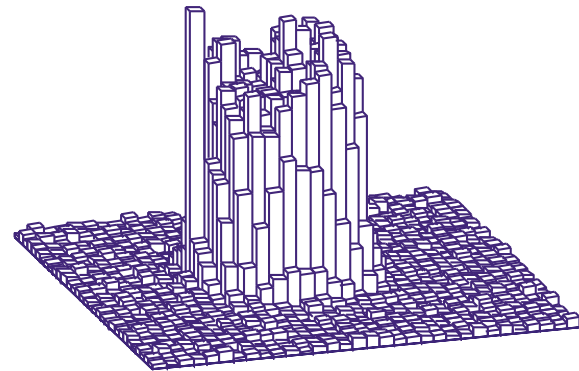

(a) Example of raw image, composed of source star, background, readout and Poissonian noises, and impacted pixels.

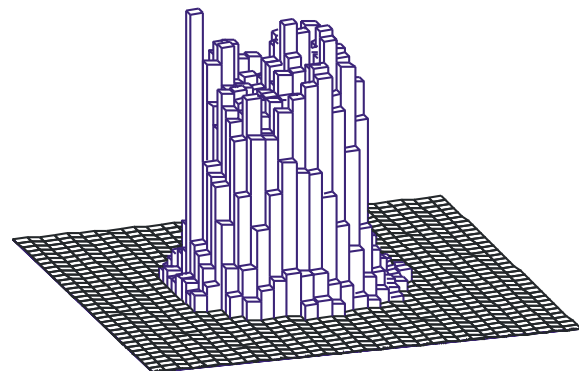

(b) The aperture technique adds up all pixels within the mask (in blue). This may subject the extracted light curve to gliches due to impacted pixels.

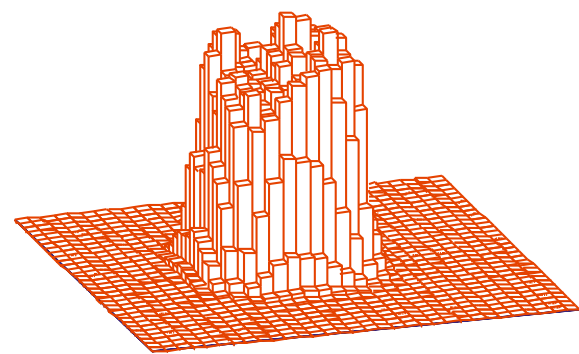

(c) The PSF-fitting technique is robust to outliers effects, but might reduce the global SNR of the photometric signal, due to its intrinsic process of fitting the acquired image to a model.

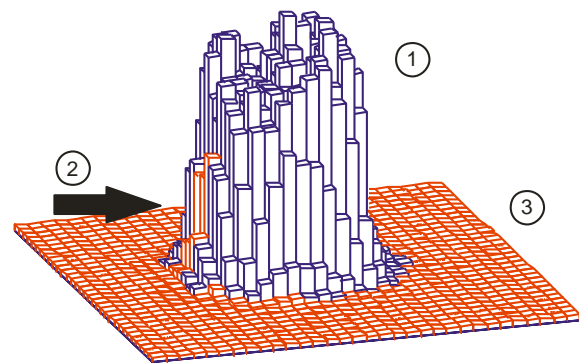

(d) The hybrid algorithm takes into account (1, blue) the high SNR pixels from observed star image, $(2$, red $)$ the replaced outliers, and $(3$, red $)$ the low uncertainty pixels from the PSF wings. The final light curve is derived from the sequence of hybrid images.

Fig. 7. Major characteristics and differences of each photometric reduction technique: (b) Aperture, (c) PSF-fitting and (d) Hybrid.

So, the starting point to the hybrid algorithm is to use all the pixels in the aperture mask defined for a specific star (or set of stars). The assumption here is that their individual high SNR will contribute to the overall SNR of the total light flux from the star.

Eq.(1) holds true under the assumption that each pixel presents pure Gaussian, mutually independent, additive noise. This is not true for real star images, since the Poissonian shot noise is closely related to the pixel intensity, which in its turn, is dependent on the architectural shape of the telescope. The main effect of this assumption is that low-intensity pixels, particularly those corresponding to the star wings are penalized, as their much lower statistical uncertainty is not properly taken into account.

Aperture photometry either does not consider wing pixels' values, or in doing so, has to cope with the implied lower SNR (real values of wing pixels present low SNR compared to central pixels of the star image). The proposed hybrid algorithm, however, instead of not considering these values, takes into account their corresponding PSF-fitted values, which actually present lower statistical uncertainty. Therefore, it is possible to exploit faint, but free-of-noise, modeled wing pixels, which contributes to the accuracy of the stellar light curves acquired with the CoRoT Telescope. Moreover, the hybrid algorithm, as the PSF-fitting technique, detects and rejects eventual impacted pixels, replacing them by their corresponding PSF-fitted values.

The main idea behind the algorithm is to exploit real acquired data where the SNR is high (central pixels), while also making use of the low uncertainty PSF wing pixels. Outlier pixels are also replaced by their fitted values, increasing the global photometric SNR. Fig.7 illustrates each component of the hybrid image.

\section{RESULTS}

The hybrid algorithm was applied to simulated data for the CoRoT telescope. The evaluations accounted for the SAA effects on the CCDs, over a stable point source of light. Therefore, any periodic variation in a real light curve would be likely to be generated from stellar seismology activities, or from exoplanetary transits, if the disturbance is achromatic. The results are compared to those of the aperture and PSFfitting techniques. The effects of the SAA on the acquired images reflect on their extracted light curve as a Gaussian-like increase in the noise component over the one-orbit period of time.

Note that, in Fig. 8, even if the aperture mask (green) is optimal in the sense that it maximizes the photometric SNR, it presents the most noisy light curve of the three evaluated methods when the satellite crosses the SAA (threshold photometry is not evaluated, since it is not used in CoRoT).

On the other hand, PSF-fitting algorithm (blue) is the one that presents the smallest photometric standard deviation, that is, the most stable light flux. Nevertheless, even this technique can not extract accurate data when the instrument is crossing the SAA; its use increases the operational duty cycle of the telescope up to $97 \%$ [1]. However, this technique intrinsically "smoothens" the signal, due to its model-fitting algorithm, which acts as a real low-pass filter in the frequency domain. Too faint light flux disturbances from the star might be filtered in this case. 
Finally, the hybrid algorithm presents an alternative technique, which provides both the increased operational duty cycle, as well as the high photometric signal from the star, presenting a relatively low standard deviation value, compared to the Aperture technique when crossing the SAA (TABLE I).

TABLE I shows the statistical comparison for the three approached techniques, comparing the standard deviation of the light curves both when the satellite is inside and outside the region of SAA's influence.

Fig. 9 illustrates another simulation, where the target star's light curve varies in time, indicating stellar seismology activities.

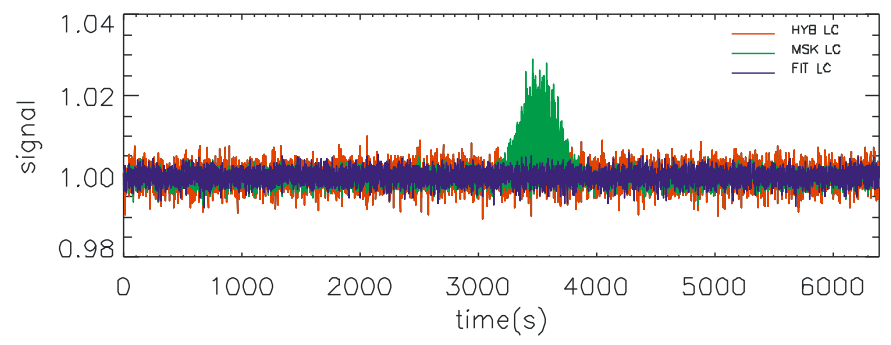

Fig. 8. Normalized light curves extracted from a simulated star: aperture (green), PSF-fitting (blue) and hybrid algorithm (red).

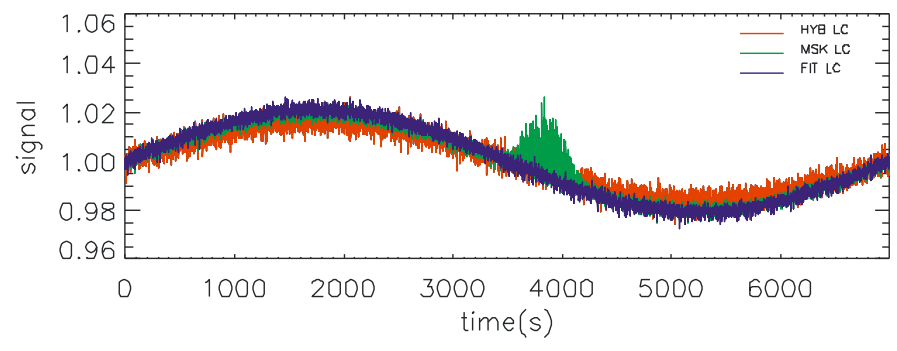

Fig. 9. Normalized light curves extracted from a simulated active star, where jitter, noise and impacts within SAA might compromise the scientific data analysis. Aperture (green), PSF-fitting (blue) and hybrid (red) algorithms' results are shown.

\section{CONCLUSIONS}

In a low-Earth-orbital environment, any spacecraft is subjected to the effects of the SAA. In the case of the CoRoT satellite, not only the use of radiation-hard components is crucial, but also effective photometric algorithms are necessary in order to enable robust and accurate data exploitation.

The aperture photometry performed onboard is optimal in scenarios where the attitude control is highly efficient and the environment is "free" from impacts of charged particles. It is computationally easy to implement, while presenting most of the time a very good photometric performance (approximately $7 \%$ of the reduced data is flagged as outliers due to radiation impacts).

The PSF-fitting photometry performs better for faint stars, in the presence of satellite attitude jitter or stray light. It is run by the ground-segment team, due to its higher computational costs, and increases the duty cycle in $3 \%$.

The hybrid algorithm combines the benefits from both techniques, associating the advantage of an empirical high- resolution instrumental PSF to the high SNR obtained in the onboard aperture photometry.

This work shows that although a significant increase in the duty cycle is not achieved (compared to the PSF-fitting methodology), the light curves obtained with the hybrid algorithm present a more realistic standard deviation while dealing with outlier pixels as well as the PSF-fitting algorithm, as a result of the use of information from both the high SNR pixels in the center of the star and the low-uncertainty pixels from its edges. Besides, outlier pixels due to radiation are replaced by empirical expected values from the PSF itself, thus eliminating impacted and/or high uncertainty pixels and increasing the global SNR, allowing gains in photometric precision.

Launched in December 27th, 2006, and with a nominal lifetime of 2.5 years, the CoRoT mission has been extended until March 31st, 2013, which will allow the hybrid algorithm to be performed in data still to be acquired and processed.

TABLE I. STATISTICAL COMPARISON FOR THE THREE TECHNIQUES

\begin{tabular}{|c|c|c|c|}
\hline Method & $\operatorname{Mean}\left(\boldsymbol{N}^{*}\right)$ & $\boldsymbol{\sigma}\left(\boldsymbol{N}^{*}\right) / \boldsymbol{\sigma}_{\boldsymbol{0}}$ & $\boldsymbol{\sigma}\left(\boldsymbol{N}^{*}\right)_{S A A} / \boldsymbol{\sigma}_{\boldsymbol{0}}$ \\
\hline Aperture & 1.0 & 1.000 & 3.457 \\
\hline PSF-Fitting & 1.0 & 1.027 & 1.061 \\
\hline Hybrid & 1.0 & 1.654 & 1.750 \\
\hline
\end{tabular}

$\operatorname{Mean}\left(N^{*}\right)$ : normalized light curve mean value. $\sigma\left(N^{*}\right)$ : standard deviation value outside the SAA. $\sigma\left(N^{*}\right)_{S A A}$ : standard deviation value inside the SAA. $\sigma_{0}$ : standard deviation outside the SAA in aperture photometry, $1.603 \times 10^{-3}$.

\section{AKNOWLEDGEMENTS}

The authors would like to thank Dr. Michel Auvergne and Dr. Janot-Pacheco, for the opportunity of research in the aerospacial imaging field, and for the technical assistance. The present work was partially funded by FAPESP (State of Sao Paulo Research Foundation, grant \# 07/56912-7).

\section{REFERENCES}

[1] M. Auvergne et al, The CoRoT Satellite in flight: description and performance. Astronomy \& Astrophysics, vol. 506, pp. 411-424, 2009.

[2] V. Lapeyrere, Étalonnage des détecteurs de CoRoT: adaptation aux besoins spécifiques de la mission. Thesis, Université de Paris VI, 2006.

[3] F. Fialho et al., Jitter Correction Algorithms for the CoRoT Satellite Mission: Validation with Test-Bench Data and MOST On-Orbit Photometry. PASP, vol. 119, pp. 337-346, 2006.

[4] S.B. Howell, Two-dimensional aperture photometry: signal-to-noise ratio of point-source observations and optimal data-extraction techniques. PASP, vol. 101, pp.616-622, 1989.

[5] L. Pinheiro da Silva et al., Estimation of a super-resolved PSF for the data reduction of undersampled stellar observations. Astronomy \& Astrophysics, vol. 452, pp. 363-369, 2006.

[6] M. Ben-Ezra, S. K. Nayar, Motion-Based Motion Deblurring. IEEE Transactions on Pattern Analysis and Machine Intelligence, vol. 26, pp. 689-698, 2004. 